\title{
Metal-insulator transitions in cyclotron resonance of periodic nanostructures due to avoided band crossings
}

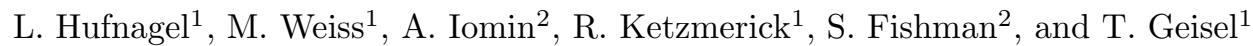 \\ ${ }^{1}$ Max-Planck-Institut für Strömungsforschung and Institut für Nichtlineare Dynamik der Universität Göttingen, \\ Bunsenstr. 10, 37073 Göttingen, Germany \\ ${ }^{2}$ Department of Physics, Technion, Haifa 32000, Israel
}

\begin{abstract}
A recently found metal-insulator transition in a model for cyclotron resonance in a two-dimensional periodic potential is investigated by means of spectral properties of the time evolution operator. The previously found dynamical signatures of the transition are explained in terms of avoided band crossings due to the change of the external electric field. The occurrence of a cross-like transport is predicted and numerically confirmed.
\end{abstract}

\section{INTRODUCTION}

Since the pioneering work of Bloch 1 and Landaul the investigation of spectral properties and transport features of Bloch electrons in magnetic field ${ }^{3}$ have attracted much attention. While infinitely degenerate Landau levels are found for free electrons in a static magnetic field, a spatially periodic potential without field yields a band structure in the spectrum. The general situation of a periodic potential and a magnetic field is still a matter of intensive research 1 . A particular simple realization for a two-dimensional (2D) periodic potential is, e.g., given by

$$
V(\mathbf{r})=V_{x} \cos \left(\frac{2 \pi x}{a}\right)+V_{y} \cos \left(\frac{2 \pi y}{b}\right)
$$

where $a, b$ are the periods in the corresponding directions. In a one-band approximation this potential leads to the well-known Harper modell, where a metalinsulator transition (MIT) is observed upon changing the modulation amplitudes from $V_{y}>V_{x}$ to $V_{x}>V_{y}$ l. At the critical point, i.e., $V_{x}=V_{\text {the spectrum and the }}$ eigenfunctions aramultifractalsid and give rise to anomalous diffusion 11,13.12. The one-band Harper model, however, has an integrable classical limit, whereas Bloch electrons in a magnetic field show chaotic dynamics in the classical limit. In quantum mechanics this is reflected in the coupling of Landau bands. This coupling leads to avoided band crossings, which induce an unusual transport phenomenon: For $V_{x} \neq V_{y}$ electrons show ballistic transport in the direction of strong potential modulation and localization in the direction of weaker modulation 7 .

Analogous phenomena can be found in driven systems with a chaotic classical limit even within the one-band approximation. A well studied example is the kicked Harper model (KHM)14 19. For small kicking strength its behavior is analogous to the Harper model 15 , while for increasing kicking strength avoided band crossings due to the classical non-integrability induce a variety of MITs 18 . Recently, it was demonstrated that a variety of kicked models, including the KHM, can be realized for electrons on a lattice in the presence of a magnetic field driven by a smooth electric field 20. The effective kicking is a result of a resonant interaction between the electronic motion and the driving field. These models correspond to cyclotron resomance experiments in antidot arrays and in organic metals 2021. By investigating the dynamics, a localization-delocalization transition induced by the amplitude of the electric field was observed 21 and remained unexplained so far.

In this paper we investigate these dynamical properties in terms of spectral properties in a wide range of driving strengths. We show that the previously found localization-delocalization transition is a consequence of avoided band crossings due to the change of the driving field strength. Moreover, the spectral analysis predicts a cross-like ballistic transport, which is numerically confirmed. We start in Section II by briefly introducing the model and explain the numerical approach in Section III. In Section IV we present our main results, which give a clear picture for the MIT in terms of the spectrum. We finally summarize our findings in Section V.

\section{THE MODEL}

The one particle Hamiltonian, describing the cyclotron resonance of an electron in the 2D periodic potential $V(\mathbf{r})$ of Eq. (11) can be written in the following form:

$$
\mathcal{H}=\frac{1}{2 m^{*}}(\mathbf{p}-e \mathbf{A}(\mathbf{r}, t))^{2}+V(\mathbf{r}),
$$

where $\mathbf{p}=\left(p_{x}, p_{y}\right)$ is the momentum of an electron with effective mass $m^{*}$ and charge $e$ moving in the $x y$ plane perpendicular to the constant magnetic field $B$ pointing in $z$-direction. We choose the Landau gauge, which yields a vector potential $\mathbf{A}(\mathbf{r}, t)=\left(\frac{E_{x}}{\nu} \sin \nu t, x B-\right.$ $\left.\frac{E_{y}}{\nu} \cos \nu t\right)$ containing also the alternating electric field $\mathbf{E}=\left(-E_{x} \cos \nu t, E_{y} \sin \nu t\right)$ with frequency $\nu$.

Applying a gauge transformation to the Hamiltonian (2), the time dependence san be shifted to the periodic potential $V(\mathbf{r}) \rightarrow V(\mathbf{r}, t)^{21}$ : If the fields $B, E_{x}, E_{y}$ satisfy $\omega^{*} E_{y}=\nu E_{x}$ then one can rewrite the Hamiltonian as $\mathcal{H}=\mathcal{H}_{0}(\mathbf{p}, x)+V(\mathbf{r}, t)$, where $\mathcal{H}_{0}=\mathcal{H}_{0}(\mathbf{p}, x)$ 
describes the cyclotron motion with frequency $\omega^{*}=$ $e B / m^{*}$. The perturbation $V(\mathbf{r}, t)$ splits the infinitely degenerate Landau levels of $\mathcal{H}_{0}$ into a mini-band structure. For strong electric fields apd $=1$ this Hamiltonian $\mathcal{H}$ reduces to a kicked system 202

$$
\begin{aligned}
\tilde{\mathcal{H}}=L \cos p+K \sum_{n=-\infty}^{\infty} \quad\left\{\cos \left(q-\kappa_{0}\right) \delta(t-2 n+1)\right. \\
\left.+\cos \left(q+\kappa_{0}\right) \delta(t-2 n)\right\}
\end{aligned}
$$

where the canonical operators $q$ and $p$ corresponding to the spatial coordinates $x, y$ satisfy $[p, q]=-i 2 \pi \hbar_{\mathrm{eff}}$ and $\kappa_{0}=\left(2 \pi e E_{y} /\left(b m^{*}\right)-\pi / 4\right) \bmod 2 \pi ; L, K$ denote the rescaled amplitudes of the potential (11). The effective Planck constant $\hbar_{\mathrm{eff}}=\hbar /(a b e B)$, corresponds to the inverse number of magnetic flux quanta through a unit cell $a \times b$. The system described by Eq. (3) has a chaotic classical limit20 and for $\kappa_{0}=0$ reduces to the KHM.

\section{NUMERICAL APPROACH}

The time evolution operator for one period corresponding to (3) reads

$$
\begin{aligned}
\mathcal{U}= & \exp \left(-\frac{i L}{\hbar_{\mathrm{eff}}} \cos p\right) \exp \left(-\frac{i K}{\hbar_{\mathrm{eff}}} \cos \left(q-\kappa_{0}\right)\right) \\
& \times \exp \left(-\frac{i L}{\hbar_{\mathrm{eff}}} \cos p\right) \exp \left(-\frac{i K}{\hbar_{\mathrm{eff}}} \cos \left(q+\kappa_{0}\right)\right) .
\end{aligned}
$$

In order to obtain its spectrum, one has to approximate the irrational effective Planck constant by a rational approximant, $\hbar_{\mathrm{eff}}=2 \pi M / N$. Following standard techniques for kicked systems 22 , this leads to a $N \times N$-matrix for the evolution operator $\mathcal{U}$, which depends on two Bloch phases $\theta_{q}, \theta_{p} \in[0,2 \pi]$. These Bloch phases arise from periodic boundary conditions in the $q$ - and $p$-direction, respectively. Diagonalization of $\mathcal{U}$ for all pairs $\left(\theta_{q}, \theta_{p}\right)$ gives its spectrum. Keeping $\theta_{q}$ fixed and varying $\theta_{p}$ yields a part of this spectrum, from now on referred to as the " $p$-spectrum". Eigenfunctions, which are localized in $p$ direction will be associated with very narrow bands in the $p$-spectrum, whereas extended states give rise to broad bands. Since for higher approximants of $\hbar_{\mathrm{eff}}$ the width of the narrow bands decreases exponentially, we will call them levels. In an analogous way one can define the " $q$ spectrum", which reflects the properties of the eigenfunctions in $q$-direction. Figure 1 shows a $q$ - and $p$-spectrum, where one observes broad bands and levels.

In order to investigate dynamics, we will study the spreading of wave packets, initially $\delta$-localized in either $q$ - or $p$-direction by determining the corresponding variances $M_{q}(t)$ and $M_{p}(t)$. For the spreading in $p$-direction we use periodic boundary conditions in $q$-direction after one unit cell, giving a discrete lattice in momentum space with spacing $\hbar_{\mathrm{eff}}$. An analogous construction is used for the spreading in $q$-direction. When considering transport in phase space in both directions, we use periodic boundary conditions after $r$ unit cells in $q$-direction, which gives $r$ different Bloch phases $\theta_{q}=2 \pi j / r(j=0, \ldots, r-1)$. The complete wave packet is then obtained by combining the wave packets for each Bloch phase and it is defined on a grid with spacing $\hbar_{\text {eff }} / r$ in momentum space.

\section{RESULTS}

We will study the spectrum of Eq. (四) as a function of $\kappa_{0}$ and its consequences for dynamical properties. If particular, we will focus on the parameters used in Ref.21 and show, that they reflect the generic behavior of the model (3)

In Ref.21 for $K=L=5$ and $\hbar_{\text {eff }}=2 \pi /\left(7+\sigma_{g}\right)$, where $\sigma_{g}=(\sqrt{5}-1) / 2$ denotes the golden mean, a transition from diffusive to ballistic dynamics was found, when $\kappa_{0}$ was varied from 0 to $\pi \sigma_{g}$ (cf. Fig. 1 of Ref.21). In Fig. 1 we present the $q$ - and $p$-spectrum for one period of $\kappa_{0}$. Apparently, the duality of $q$ - and $p$-spectrum is conserved for all $\kappa_{0}$, i.e., the $q$-spectrum shows wide bands, where the $p$-spectrum is level-like and vice versa.

For $\kappa_{0}=0$ the variances increase almost linearly in both $q$ - and $p$-direction, corresponding to diffusive spreading in either direction (top of Fig. 2a). This well-known behavior of the KHM is a direct consequence of the mpltifractal nature of spectrum and eigenfunctions $14-16$.

For $\kappa_{0}=\pi \sigma_{g}$ bands occur in the $p$-spectrum explaining the ballistic transport in $p$-direction observed in Ref.21. There are, however, also levels, which are related to bands in the $q$-spectrum, predicting a ballistic spreading in $q$-direction. This is confirmed in Fig. 2 $\mathrm{b}$. All eigenstates are extended in either of the directions and localized in the other one. A typical initial wave packet will excite both types of eigenfunctions. The part of the wave packet consiting of eigenfunctions extended in the $q$ direction gives ballistic transport in the $q$-direction. The other part, which is a superposition of eigenfunctions extended in the $p$-direction, yields ballistic transport in the $p$-direction. Therefore one finds a superposition of ballistic transport in $q$ - and $p$-direction, namely a cross-like transport (bottom of Fig. 2 $\mathrm{b}$ ). The cross-like form of the wave packet is clearly different from the isotropic form for $\kappa_{0}=0$ (Fig. 2a). There are several other transport features one can observe from the Husimi plot in Fig. 2b. The weight of the wave packet spreading ballistically along the $p$-direction is much bigger than the corresponding weight for the $q$-direction. This is a signature of the spectrum, namely that there are more bands in the $p$-spectrum than there are bands in the $q$-spectrum at $\kappa_{0}=\pi \sigma_{g}$, as can be seen in Fig. 1. It should be noted, that the velocities in $p$ - and $q$-direction (which can be inferred from the variances $M_{p}(t)$ and $\left.M_{q}(t)\right)$ are in general not related to the number of bands but are determined by the band widths. 
Now we will turn away from the symmetry line $K=L$ and focus on the parameters $K=2, L=4, \hbar_{\mathrm{eff}}=$ $2 \pi /\left(7+\sigma_{g}\right)$. In Fig. 3 we show the $q$ - and $p$-spectrum as a function of $\kappa_{0}$, which again appears to be dual. For the $\mathrm{KHM}\left(\kappa_{0}=0\right)$ the $q$-spectrum shows broad bands, whereas the $p$-spectrum is level-like, indicating transport in $q$-direction, but localization in $p$-direction 14. This is confirmed by the dynamics (Fig. A a). Variation of $\kappa_{0}$ induces avoided band crossings leading to the occurrence of bands in the $p$-spectrum (see magnification in Fig. 3). This is associated with a localization-delegalization transition in $p$-direction as observed in Ref.21. As there are still bands in the $q$-spectrum one again finds the generic cross-like transport (Fig. \$b). Here, the weight of the wave packet spreading along the $q$-direction is bigger than the corresponding weight in $p$-direction, as is implied by the much larger number of bands in the $q$-spectrum (Fig. 3). Another characteristic of the wave packet is the increased localization length in $p$-direction of the part of the wave packet spreading in $q$-direction compared to Fig. 4a. This is due to the increased localization length of the eigenstates corresponding to the levels in the $p$ spectrum. This change in the localization length can be explained by avoided band crossings 18 .

\section{SUMMARY}

In conclusion, we have shown here, that the previously found MIT in cyclotron resonance in a 2D-periodic potential in the presence of a magnetic field can be fully understood by investigating the spectrum of the corresponding time evolution operator. We have presented numerical evidence, that the duality of the spectra is conserved under changes of the driving strength. We were able to explain the previously observed dynamics21, which has shown a transition from diffusive to ballistic transport as well as a transition from localization to ballistic transport in the $p$-direction. Furthermore, the spectra allowed us to predict the transport behavior in $q$-direction leading to a cross-like transport. These predictions were also confirmed numerically. We would like to mention, that cross-like transport is generic for systems with dual spectra and a chaotic classical limit.

The observed transport phenomena in phase space of the effective Hamiltonian (3) correspond to analogous transport phenomena in the $x y$-plane. The required conditions for realization of the observed MIT may well be achieved in experiments on cyclotron resonance 23 of a $2 \mathrm{D}$ electron gas embedded in lateral superlattices fabricated on GaAs heterostructures 24 .

This research was supported by the Niedersachsen Ministry of Science. A.I. and S.F. were also supported by the Israel Science Foundation founded by the Israel Academy of Sciences and Humanities. A.I. thanks Prof. T. Geisel for his hospitality at the MPI für Strömungsforschung.
${ }^{1}$ F. Bloch, Z. Phys. 52, 555 (1929).

${ }^{2}$ L. Landau, Z. Phys. 64, 629 (1930).

${ }^{3}$ R. Peierls, Z. Phys. 80, 763 (1933).

${ }^{4}$ C. Albrecht et al., Phys. Rev. Lett. 93, 2234 (1999).

${ }^{5}$ T. Schlösser et al., Europhys. Lett. 33, 683 (1996).

${ }^{6}$ A. Barelli, J. Bellisard, and F. Claro, Phys. Rev. Lett. 83, 5082 (1999).

${ }^{7}$ R. Ketzmerick, K. Kruse, D. Springsguth, and T. Geisel, Phys. Rev. Lett. 84, 2929 (2000).

${ }^{8}$ P.G. Harper, Proc. Phys. Soc. Lond. A68, 874 (1955).

${ }^{9}$ S. Aubry and G. André, in Proc. of the Israel Phys. Soc., edited by C. G. Kuper, v. 3, 133 (Hilger, Bristol, 1979).

${ }^{10}$ D. R. Hofstadter, Phys. Rev. B 14, 2239 (1976); C. Tang and M. Kohmoto, Phys. Rev. B 34, 2041 (1986).

${ }^{11}$ H. Hiramoto and S. Abe, J. Phys. Soc. Jpn. 57, 230 and 1365 (1988).

12 T. Geisel, R. Ketzmerick, and G. Petschel, Phys. Rev. Lett. 66, 1651 (1991).

${ }^{13}$ R. Ketzmerick, K. Kruse, S. Kraut, and T. Geisel, Phys. Rev. Lett. 79, 1959 (1997).

${ }^{14}$ R. Lima and D. Shepelyansky, Phys. Rev. Lett. 67, 1377 (1991).

15 T. Geisel, R. Ketzmerick, and G. Petschel, Phys. Rev. Lett. 67, 3635 (1991); 69, 695 (1992).

${ }^{16}$ R. Artuso, G. Casati, and D. Shepelyansky, Phys. Rev. Lett. 68, 3826 (1992); R. Artuso et al., Phys. Rev. Lett. 69, 3302 (1992).

17 I. Dana, Phys. Rev. Lett. 73, 1609 (1994); Phys. Lett. A 197, 413 (1995).

${ }^{18}$ R. Ketzmerick, K. Kruse, and T. Geisel, Phys. Rev. Lett. 80, 137 (1998).

19 A. Iomin, G.M. Zaslavsky, Phys. Rev. E 60, 7580 (1999); Chaos 10, 147 (2000).

${ }^{20}$ A. Iomin and S. Fishman, Phys. Rev. Lett. 81, 1921 (1998); Physica D 131, 170 (1999).

${ }^{21}$ A. Iomin and S. Fishman, Phys. Rev. B 61, 2085 (2000).

22 S.-J. Chang, K.-J. Shi, Phys. Rev. A 34, 7 (1986).

${ }^{23}$ E. Vasiliadou, et al., Phys. Rev. B 52, R8658 (1995).

${ }^{24}$ For a review, see W. Hansen, U. Merkt, and J.P. Kotthaus, in Nanostructured Systems, edited by M. Reed, Semiconductors and Semimetals Vol. 35 (Academic, San Diego, 1992), p. 279.

FIG. 1. The $p$-spectrum (top) and $q$-spectrum (bottom) of the evolution operator (4) for $K=L=5$ vs. $\kappa_{0}$ (one period $[-\pi / 2, \pi / 2]$ is shown). The approximant for the irrational effective Planck constant is $\hbar_{\mathrm{eff}}=2 \pi \cdot 34 / 259$. Arrows denote the values $\kappa_{0}=0$ and $\kappa_{0}=\pi \sigma_{g}$ used in Fig. For $\kappa_{0}=0$ Eq. (3) reduces to the KHM and the spectrum is multifractal, while for $\kappa_{0} \neq 0$ it consists of bands and levels. The spectra appear to be dual to each other, since broad bands in one of them are associated with levels in the other. 
FIG. 2. (a) Variances $M_{q}(t), M_{p}(t)$ and the Husimi plot of a 2D wave packet after 100 kicks (one point per unit cell and $r=89$ cells in each direction) for $K=L=5$, $\hbar_{\mathrm{eff}}=2 \pi /\left(7+\sigma_{g}\right), \kappa_{0}=0$. (b) same for $\kappa_{0}=\pi \sigma_{g}$. In the first case, the variances show a diffusive-like behavior while the wave packet spreads ballistically in both directions in the second case. This is also reflected in the Husimi plots, where one observes that the wave packet spreads isotropically in phase space in (a) and shows a cross-like spreading in (b).

FIG. 3. Same as in Fig. 1 for $K=2, L=4$. For $\kappa_{0}=0$ the $p$-spectrum consists of levels only and the $q$-spectrum shows bands. Magnifications $\left(\hbar_{\mathrm{eff}}=2 \pi 55 / 419\right)$ show part of the spectra around $\kappa_{0}=\pi \sigma_{g}$, where one finds bands in the $p$-spectrum and levels in the $q$-spectrum. Duality again seems to be conserved.

FIG. 4. Same as in Fig. 1 for $K=2, L=4$. (a) Ballistic spreading in $q$-direction and localization in $p$-direction is observed. (b) Ballistic spreading in both directions leads to a cross-like transport. 
This figure "fig1.gif" is available in "gif" format from: http://arxiv.org/ps/cond-mat/0008212v1 
This figure "fig2.gif" is available in "gif" format from: http://arxiv.org/ps/cond-mat/0008212v1 
This figure "fig3.gif" is available in "gif" format from: http://arxiv.org/ps/cond-mat/0008212v1 
This figure "fig4.gif" is available in "gif" format from: http://arxiv.org/ps/cond-mat/0008212v1 